\title{
Joint Interleaver and LDPC Code Parameter Design for Beyond G.fast Systems
}

\author{
Cornelius T. Healy*, Anas F. Al Rawi ${ }^{\dagger \ddagger}$, and Charalampos C. Tsimenidis* \\ ${ }^{*}$ Newcastle University, School of Electrical and Electronic Engineering \\ Newcastle upon Tyne, NE1 7RU, England, UK \\ ${ }^{\dagger}$ BT labs, Research and Design, BT plc, Adastral Park, Martlesham Heath, IP5 3RE, UK \\ $\ddagger$ Cavendish Laboratory, University of Cambridge, Cambridge, CB3 0HE, UK
}

\begin{abstract}
To achieve increased capacity over legacy twistedpair copper, standards have considered use of increasingly high frequencies. The most recent digital subscriber line (DSL) standard, G.fast, carried forward the forward error correction scheme from the previous standards, namely the concatenated Reed Solomon and multi-dimensional trellis coded modulation scheme. In this work, a novel joint code and interleaver optimisation for low-density parity-check (LDPC) coded multicarrier systems with variable bit loading is proposed. This optimisation exploits the effects of both the channel and the modulation scheme on the soft information available at the LDPC decoder, through use of extrinsic information transfer (EXIT) analysis. The performance of the proposed scheme is demonstrated for the discrete multi-tone system operating on a twisted-pair channel.

Index Terms-G.fast, Forward error correction, LDPC
\end{abstract}

\section{INTRODUCTION}

The hybrid fibre-copper network has presented a costeffective route to continued fibre proliferation. Using the existing copper drop to the customer premises equipment (CPE) from a nearby distribution point (DP) avoids the disproportionately expensive installation of fibre to the home [1] [2]. As the length of the copper drop has steadily been shortened, the capacity of the link has increased, as exploited in the recent G.fast standard [3]. On the shorter twistedwire pairs, operation can be extended to higher frequency bands, provided techniques are employed to overcome the increasingly challenging channel conditions.

In Fig. 1, the direct and indirect (electromagnetic coupling) paths are illustrated for a twisted-pair binder. Near-end crosstalk (NEXT) acts as a limiting interferer due to its large magnitude with respect to the incoming direct path, and must be avoided by use of time- or frequency-division duplexing (TDD or FDD), or by the use of advanced techniques for fullduplex operation as in [4]. At the far end, far-end cross-talk (FEXT) can be cancelled through vectoring [5].

In this work, a single line under TDD operation is considered, i.e., both NEXT and FEXT are absent. Forward error correction and coded modulation schemes are then investigated under the effects of the direct channel loss. In particular, the modern LDPC-based bit-interleaved coded modulation (BICM) scheme [6] [7] is applied to the DSL system under consideration, and through EXIT-based analysis [8] the parameters of both the LDPC code and the BICM scheme are optimised for both channel and system. The design is based on the developed understanding of the equivalent binary-input continuous-output channels of the DSL system, as observed by the LDPC code.

This builds on prior work in the literature using EXIT analysis for coded modulation, and the work on the unequal error protection (UEP) [9] intrinsically offered by the discrete QAM constellations [10] [11].

The rest of this paper is organised as follows: Section II describes the system model formally, and provides preliminaries for the following sections. Section III details the proposed approach. In Section IV the simulation study supports the proposed work, while Section V offers concluding remarks.

\section{System Model ANd PReliminaries}

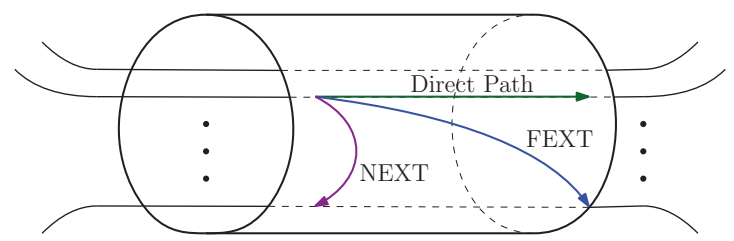

Fig. 1. The path loss and electromagnetic coupling of a twisted-pair binder.

\section{A. The DSL Channel}

As mentioned in Section I, in this work the DSL system with a single pair only from distribution point to customer premises is considered, under TDD operation. Thus, both NEXT and FEXT are absent, allowing the effect of the direct path channel loss on the coding scheme to be investigated. The channel model in this case becomes:

$$
y_{k}=h_{k} P_{k} x_{k}+n_{k}=h_{k} u_{k}+n_{k},
$$

where $h_{k}$ is the channel attenuation on DMT tone $k, x_{k}$ is the QAM symbol to be transmitted and $P_{k}$ is the transmit power at tone $k, u_{k}$ is the transmitted symbol, and $n_{k}$ is the additive white Gaussian noise (AWGN) sample. The receiver simply applies the channel inverse as

$$
r_{k}=h_{k}^{-1} y_{k}=u_{k}+h_{k}^{-1} n_{k} .
$$

The measured channel frequency response is provided in the first sub-figure of Fig. 3 for the channel of length $100 \mathrm{~m}$. As 
will be specified in Section IV, the lines under consideration in this work are length $25 \mathrm{~m}$ and length $100 \mathrm{~m}$, with measurements provided by our partners at BT.

\section{B. Coding and BICM for the DSL Channel}

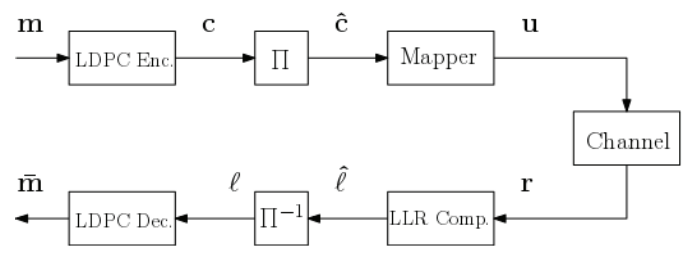

Fig. 2. The block diagram of the BICM system.

The LDPC-based BICM system is considered in this work, as shown in Fig. 2. Gray mapping is applied in the mapper block, and the LLR computation block performs the reciprocal operation to produce the soft decoder input values for each codeword bit, given knowledge of the channel attenuation and of the variance of the AWGN. QAM modulation is used throughout the paper. The mapper and LLR computation blocks operate appropriately per tone $k$ to allow varied bit loading across the tones in the DMT block.

Design of the LDPC code ensemble parameters, and of the interleaver which matches codeword bits to particular QAM bit positions on particular tones is the novel approach which is developed in this paper. Once the parameters have been designed, the code is instantiated through the construction of a code graph. In each case in this work, this is done by use of the pseudo-random Progressive Edge Growth (PEG) algorithm. The standard log-domain sum-product algorithm is used as the decoder, with a maximum of 50 iterations.

An alternative to the system in Fig. 2 is LDPC-coded modulation (LCM), which has seen much recent interest, and is under consideration for future DSL standards. The methods described in this paper may equally be applied to LCM, with changes to take into account constellation and tone mapping.

\section{FEC in the G.fast Standard}

Forward error correction in the G.fast standard [12] consists of the legacy trellis coded modulation (TCM) system [13] introducing a single bit of redundancy per two constellation symbols by means of a 4-dimensional trellis code mapping. Further error correction is supplied by an outer byte-oriented Reed-Solomon code.

In practice, near error-free operation is ensured by adaptive bit- and power-loading, taking into account the assumed coding gain of the concatenated system, $G$, and a specified gap to capacity $\Gamma$. For the single line used here, bit-loading for a desired fixed noise level $N$ and maximum modulation order $M$ is given by

$$
B_{k}=\left\lfloor\log _{2}\left(1+\mathrm{SNR}_{k}\right)\right\rfloor
$$

where $\lfloor b\rfloor$ is the greatest integer less than $b$, and $\mathrm{SNR}_{k}$ is the adjusted received SNR at tone $k$, given transmit power adjusted for gap, coding gain and PSD mask:

$$
P_{k}=\min \frac{\left(2^{M}-1\right) N}{|h|_{k}^{2}} 10^{\frac{\Gamma-G}{10}}, \operatorname{MASK}_{k},
$$

$\mathrm{MASK}_{k}$ is the PSD mask at tone $k$ of the DMT block.
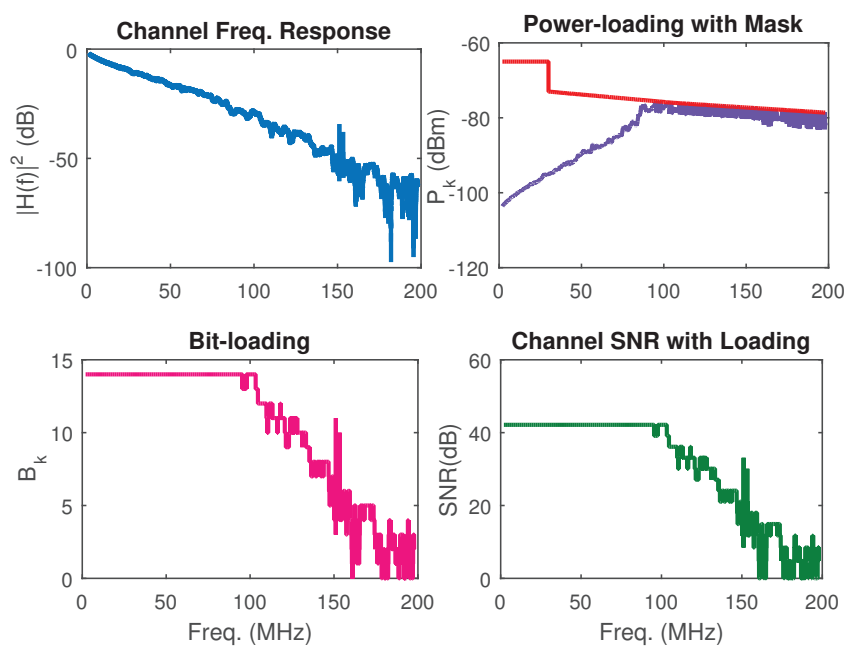

Fig. 3. The bit- and power-loading for the $100 \mathrm{~m}$ channel with maximum modulation order 14, considered in Fig. 9.

\section{EXIT Analysis}

EXIT-based analysis allows investigation of the threshold of iterative decoders by tracking the update of mutual information through successive iterations, and determining whether the code parameters and channel conditions allow convergence. For LDPC codes, each iteration is modelled as the update of two component decoders, the variable node processor and the check node processor. The variable node processor mutual information update is given by [8]:

$$
\begin{aligned}
& I_{E, V}\left(I_{A, V}, d_{v}, \frac{E_{b}}{N_{0}}, R\right)= \\
& \quad J\left(\sqrt{\left(d_{v}-1\right)\left[J^{-1}\left(I_{A, V}\right)\right]^{2}+\sigma_{c h}^{2}}\right),
\end{aligned}
$$

where the terms $I_{A, V}$ and $I_{E, V}$ are the input a priori mutual information and the extrinsic mutual information for the variable node of weight $d_{v}$, respectively. $\frac{E_{b}}{N_{0}}$ is the SNR per bit and $\mathrm{R}$ is the code rate. Furthermore, $\sigma_{c h}^{2}$ is the variance of the channel LLR and $J($.$) represents the mutual information$ given by

$$
J\left(\sigma_{c h}\right)=H(x)-H\left(x \mid L_{c h}(Y)\right),
$$

approximated numerically to allow analytical evaluation.

The inverse mutual information update for the check node component decoder is approximated by 


$$
I_{A, C} \approx 1-J\left(\frac{J^{-1}\left(1-I_{E, C}\right)}{\sqrt{d_{c}-1}}\right)
$$

where $I_{E, C}$ is the extrinsic mutual information at the check node processor and $d_{c}$ is the check node degree.

Irregular LDPC codes are described by their code parameters, namely the code rate and degree distribution pairs. The degree distribution pair is given by:

$$
\begin{aligned}
& \lambda(x)=\sum_{i=2}^{d_{v, \max }} \Lambda_{i} x^{i-1}, \\
& \rho(x)=\sum_{i=2}^{d_{c, \max }} d_{i} x^{i-1},
\end{aligned}
$$

where $\Lambda_{i}$ and $d_{i}$ are the fraction of edges connected to a variable or check node of degree $i$, respectively. For irregular LDPC code parameter design by EXIT chart, the overall compound EXIT function is given by the weighted sum of the EXIT functions of the constituent node processors of each degree. The weighting in the sum is by $\Lambda_{i}$ and $d_{i}$, respectively, so for the compound variable node processor the mutual information update is given by:

$$
I_{E, V}\left(I_{A}, \frac{E_{b}}{N_{0}}, R\right)=\sum_{i=2}^{d_{\max }} \Lambda_{i} I_{E, V}\left(I_{A}, d_{v, i}, \frac{E_{b}}{N_{0}}, R\right) .
$$

\section{E. Bit Channels of QAM Constellations}

Under QAM signalling, the bit positions offer differing levels of error protection, a fact which has previously been exploited in code design [10] [11], bit mapping [14], and in imparting UEP by design [9].

In the next section, this inherent UEP will, along with the effect of the channel and bit-loading, be exploited through EXIT-based joint parameter and interleaver design. To allow this, the probability of error of each bit position in the different QAM constellations will be required. For square $M$-QAM constellations the probability of error is given by

$$
\begin{aligned}
P_{b}(t)= & \frac{1}{\sqrt{M}} \sum_{j=0}^{\left(1-2^{-t}\right) \sqrt{M}-1}\left[( - 1 ) ^ { \lfloor \frac { j 2 ^ { t - 1 } } { \sqrt { M } } \rfloor } \left(2^{t-1}-\right.\right. \\
& \left.\left\lfloor\frac{j 2^{t-1}}{\sqrt{M}}+\frac{1}{2}\right\rfloor\right) \operatorname{erfc}\left((2 j+1) \sqrt{\left.\frac{3 \log _{2}(M) r}{2(M-1)}\right)}\right],
\end{aligned}
$$

while for cross QAM constellations the expressions for the exact probability of error of bit positions can be found in [15].

Under the assumption that the individual bit channels may be treated as AWGN channels, the equivalent noise variance for each bit channel is then found as:

$$
\sigma_{n}^{2}(t)=\frac{1}{2\left(\operatorname{erfc}^{-1}\left(2 \mathrm{P}_{b}(t)\right)\right)^{2}}
$$

Fig. 4 provides examples of bit channels $P_{b}(t)$ for 32 QAM and 1024-QAM constellations, demonstrating that the differences in error probabilities in different positions are significant, particularly at the low-to-mid SNRs relevant to the LDPC threshold.

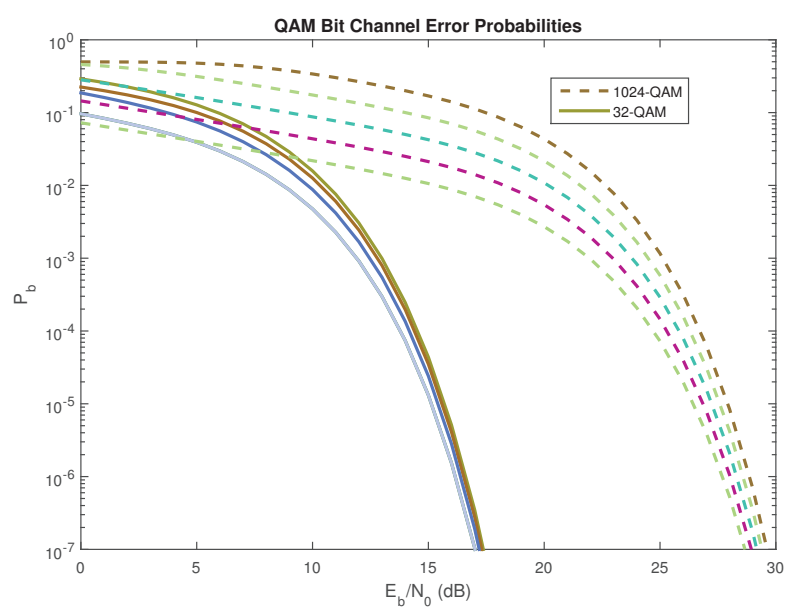

Fig. 4. The probabilities of error for the bit channels of both 32-QAM cross and 1024-QAM square constellations.

\section{Proposed Joint Code/InterleAVER Design METHOD}

The threshold benefits of irregular variable node degree in LDPC code ensembles are well established [16]. Generally, low-weight nodes offer quicker convergence, high-weight nodes increased reliability. Recognising the inherent UEP of the constellations and channel, along with this unequal decoding performance of bits in the codeword, it is possible to model the effect of both by applying the bit channel variances of (12) to the EXIT function of (5) and observing the combined effect of the bit channels on decoding performance of the system through the compound function (10).

Furthermore if a code optimisation technique is applied under these bit channel conditions, the result will be a set of code parameters for each bit channel in the system. This essentially defines the interleaver of the BICM system jointly with the LDPC code parameters.

For the joint parameter/interleaver design, a number of cases will be considered. Initially, the bit- and power-loading will be flat across the tones $k$. While this is not necessarily representative of operation under the practical DSL systems, it will allow an investigation of the benefits of the bit channel approach in isolation, removed from the effects of bit- and power-loading.

Following this, the EXIT analysis will be applied to the loaded scenario of Fig. 3.

\section{A. Illustration with Equal Bit Loading}

In this section, the effect of the channel frequency response is characterised within bands to a single representative value, in order to simplify the channel design optimisation. This provides an approximation to the true effect of the channel, which 
may be further improved upon but serves to demonstrate the potential of the proposed approach. Given the vector $\mathbf{h}$ of channel coefficients $h_{k}$, the coefficients within band $m$ of length $\frac{L}{B}$ are given by

$$
\mathbf{h}_{m}=\left[h_{a}, h_{a+1}, \cdots, h_{b-1}\right],
$$

where $a=\frac{m L}{B}$ and $b=\frac{(m+1) L}{B}$, and $L$ is the length of h. Now, the effect of the channel within the band, for the purposes of the joint design, is taken to be

$$
H_{m}^{2}=\frac{1}{B} \sum\left|\mathbf{h}_{m}\right|^{2} .
$$

Using (12), the bit channel variances on each of the $B$ bands can now be obtained as

$$
\sigma_{n}^{2}(m, t)=\frac{\sigma_{n}^{2}(t)}{H_{m}^{2}}, t=0, \cdots, K-1 ; m=0, \cdots, B-1 .
$$

The effect of these equivalent channel variances is illustrated by means of an EXIT chart for BPSK modulation $(K=1)$ and $B=5$ bands in Fig. 5, showing the significant variation in bit channel quality in the bands.

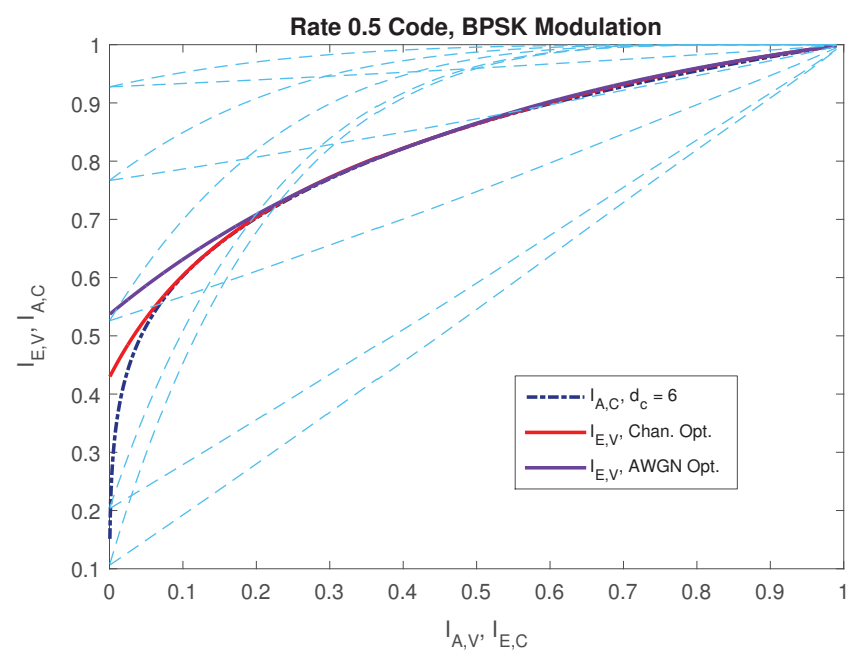

Fig. 5. The EXIT charts for the equivalent bit channels over the bands of the DSL channel, for BPSK modulation.

Once the variances of the bit channels for each band are found, analysis for a given LDPC code is straightforward, the EXIT function of (5) is evaluated for each variance (15) and combined according to their weights as in (10). The design method is a simple optimisation, with area between functions $I_{E, V}$ and $I_{A, C}$ as objective function. Care is needed only to apply constraints so that the designed code has the correct number of coded bits linked to each equivalent bit channel.

The constraints for the optimisation include the standard consistency limits placed on the degree distribution,

$$
\begin{gathered}
\sum_{i, t, m} \Lambda_{i, t, m}=1, \\
\Lambda_{i, t, m} \geq 0, \forall i, t, m,
\end{gathered}
$$

along with the constraint to ensure the solution has an open decoding trajectory, i.e., that the decoder will converge:

$$
I_{E, V}\left(I_{A, V}, \frac{E_{b}}{N_{0}}, R\right)>I_{A, C}\left(I_{E, C}, d_{c}\right)
$$

In addition to these constraints, the designed parameters should produce a code with the desired rate, and the correct proportion of variable nodes in the designed graph should be associated with each bit channel. These goals may both be achieved by applying the constraint

$$
\sum_{t, m} \frac{\Lambda_{i, t, m}}{i}=\frac{1}{K B d_{c}(1-R)}
$$

where $K$ is the total number of constellation bit channels and $B$ is the number of frequency bands considered.

Finally, the standard constraint that the number of weight-2 variable nodes is less that the number of check nodes in the graph is applied. This avoids cycles associated solely with weight-2 variable nodes [17].

$$
\sum_{i=2, t, m} \Lambda_{i, t, m}<\frac{2}{d_{c}}
$$

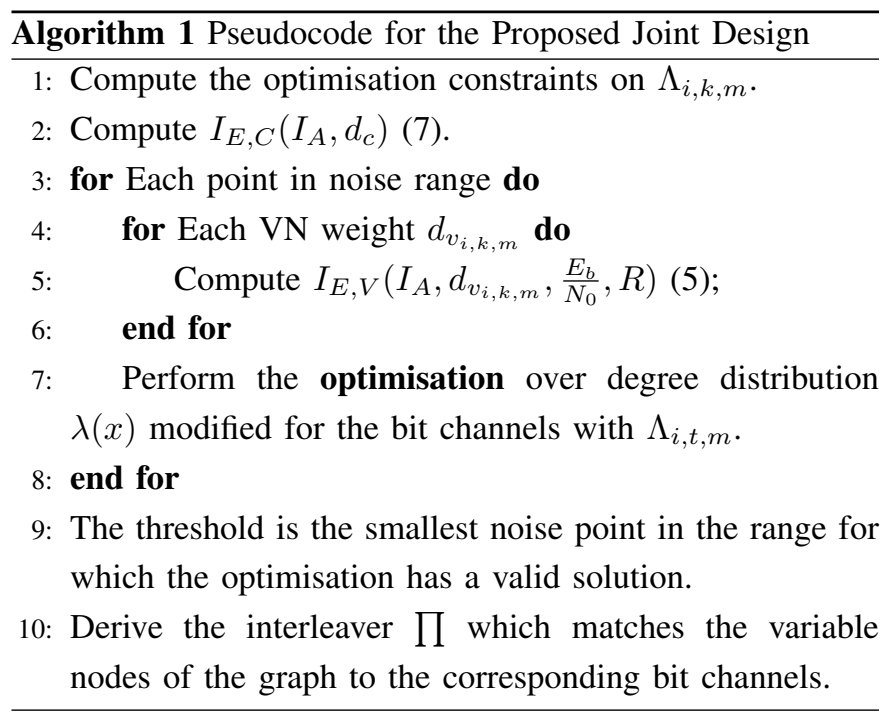

\section{B. Bit- and Power-Loading Scenario}

For the bit- and power-loaded scenario, the design follows a very similar approach to that outlined in Section III-A. However, the effect of the loading, as seen in the lower two sub-figures in Fig. 3, is to flatten the received SNR of the channels with a given modulation order. Thus, rather than taking the channel in bands of increasing tone $k$, they are taken with equal bit loading $B_{k}$. This has the added benefit that each band has an equal number of bit channels. The constraints and optimisation are applied identically to the previous section. 


\section{Simulation Study}

In this section, the simulation results for the proposed design method are presented. The results progress from the most basic (flat power-loaded BPSK) through higher-order QAM constellations with flat loading and finally to the scenario with bit- and power-loading. All LDPC graphs were constructed by PEG algorithm.

Fig. 6 presents results for the rate half LDPC code on the $25 \mathrm{~m}$ channel. The proposed design is compared to the code designed only for the AWGN channel, in terms of BER performance. Also included in the plot is the capacity limit for the discrete-input continuous-output memoryless channel (DCMC), demonstrating the capacity-approaching nature of the LDPC codes. A gain of approximately $0.5 \mathrm{~dB}$ is observed for the proposed design method.

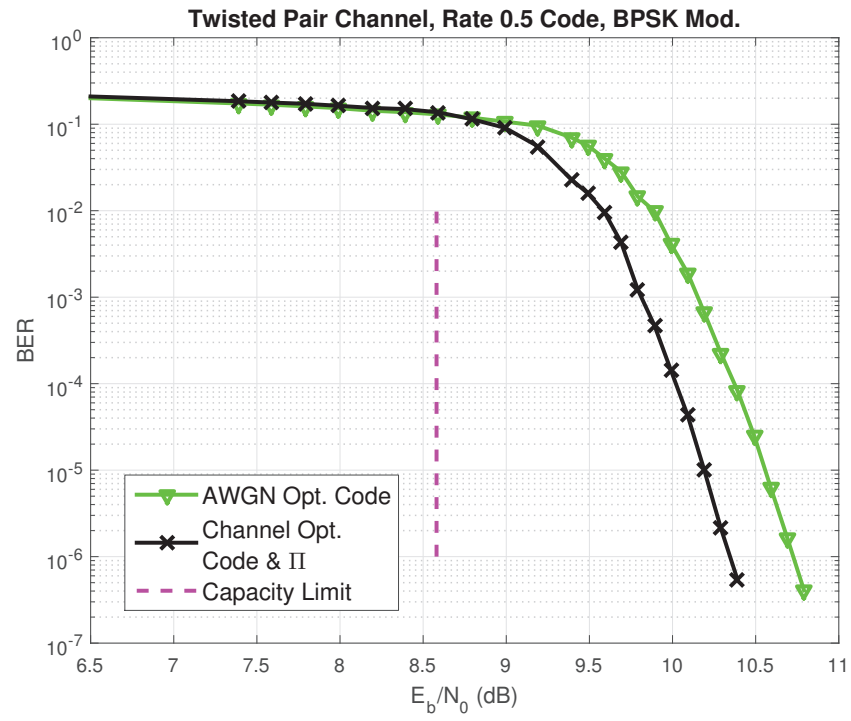

Fig. 6. Results for the BPSK modulated codes, rate 0.5 , on $25 \mathrm{~m}$ line

Fig. 7 presents the results for the QAM high order constellation, 1024-QAM on the $25 \mathrm{~m}$ channel. For this figure, the codes tested are those designed jointly for high order constellations (HOC) and the DSL channel, for HOC only on the AWGN channel, and finally the standard design for the AWGN channel only. The best-performing codes are those designed with the proposed method, while the code designed for AWGN only performs worst. The code and interleaver designed for the HOC with AWGN channel offers modest performance improvement. For the code/interleaver designed for both modulation and channel, two cases were considered. In both, the HOC specified $K=\frac{\sqrt{(M)}}{2}=5$ bit channels. In the first case, the DSL channel was divided into only 2 bands, while in the second case 5 bands were selected. For the case with the greater number of channel bands, a small but consistent improvement is observed. For these designed codes, gains of $0.2 \mathrm{~dB}$ and $0.25 \mathrm{~dB}$, respectively over the code optimised for AWGN channel only are achieved.

Fig. 8 presents results for the flat loaded scenario with 32 QAM modulation on the $25 \mathrm{~m}$ channel, showing the large gain

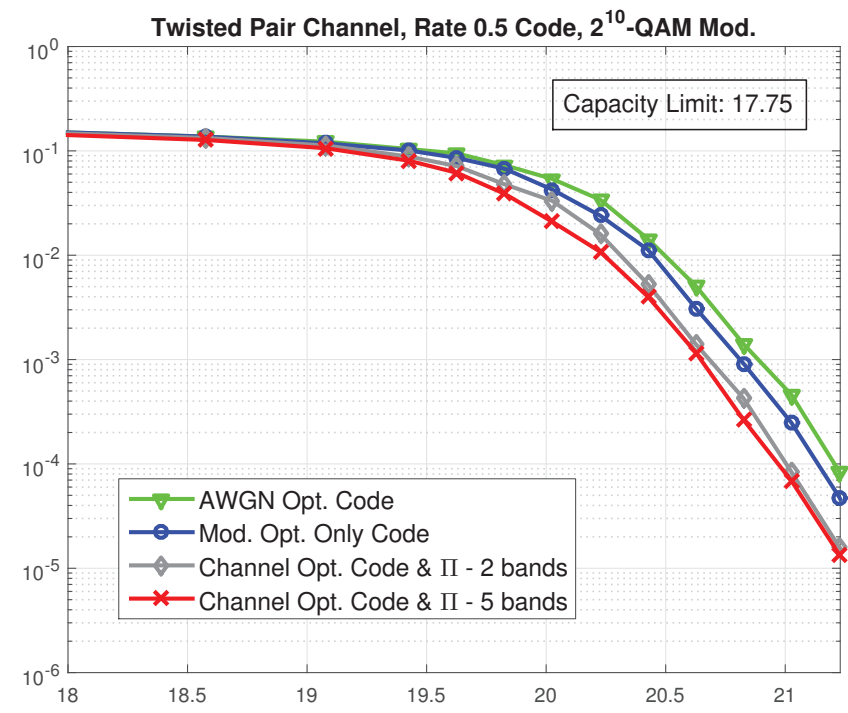

Fig. 7. Results for the 1024-QAM modulated codes, rate 0.5 , on $25 \mathrm{~m}$ line

offered by LDPC code over the coding system currently used in the G.fast standard, and additionally showing the gain of the proposed design method over the WiMAX-like codes generalised from the standard [18]. Results are presented both for short and long block length LDPC graphs, with spectral efficiency of the LDPC codes matched to that of the concatenated coding and TCM system of G.fast. For the short codes, the proposed design achieves a consistent $0.3 \mathrm{~dB}$ gain compared to standard code, and the large achieves a gain of $0.6 \mathrm{~dB}$. Gains in excess of $10 \mathrm{~dB}$ and $7 \mathrm{~dB}$ are observed compared to the RS+TCM system by the long and short LDPC codes, respectively. It should be noted that the scenario considered in Fig. 8 is not representative of normal operation in DSL systems, where the adaptive loading ensures the channel conditions observed by the coding system are significantly more benign. However, the results do demonstrate the relative strength of the modern coding scheme well.

Finally, the results for the loaded case and the $100 \mathrm{~m}$ channel of Fig. 3 are presented in Fig. 9. Here, the loading (3)(4) was set for fixed noise point $N=-150 \mathrm{dBm}$ and held constant while the noise level was then allowed to vary. By this method, relative performances of the modern and legacy coding schemes are investigated. In (4), coding gain $G$ was taken as $8 \mathrm{~dB}, \Gamma$ as $9.75 \mathrm{~dB}$, both reasonable choices in the standard practice. The maximum modulation order $M=14$. Error rates are compared at varying system noise level, and the LDPC and RS-TCM have matched spectral efficiency, with both having overall rate 0.9233 . The RS code block length is 254 bytes, including 8 bytes of redundancy. Due to the equal loading and coding rates, both systems offer equal data rate. The gain for LDPC codes over the concatenated scheme in this scenario is less dramatic than for Fig. 8, a still significant $2 \mathrm{~dB}$. Additionally the proposed design method is demonstrated, through the designed threshold value, to offer an additional $0.4 \mathrm{~dB}$ of gain over the WiMAX-like design of LDPC codes. 


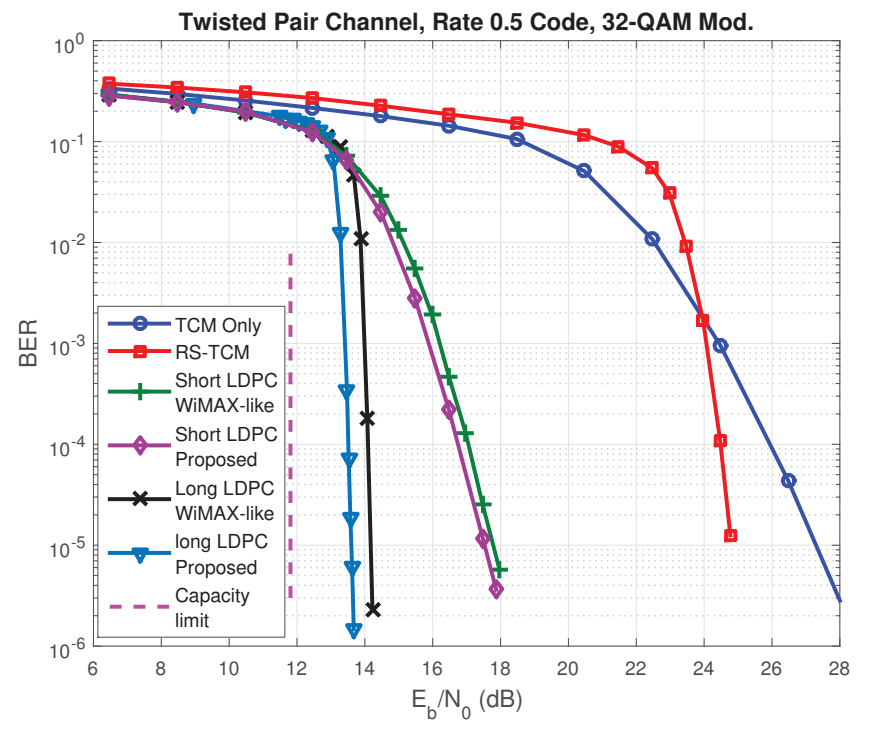

Fig. 8. Comparison of performance of an LDPC-BICM system and the RSTCM system of the G.fast standard, on the $25 \mathrm{~m}$ line.

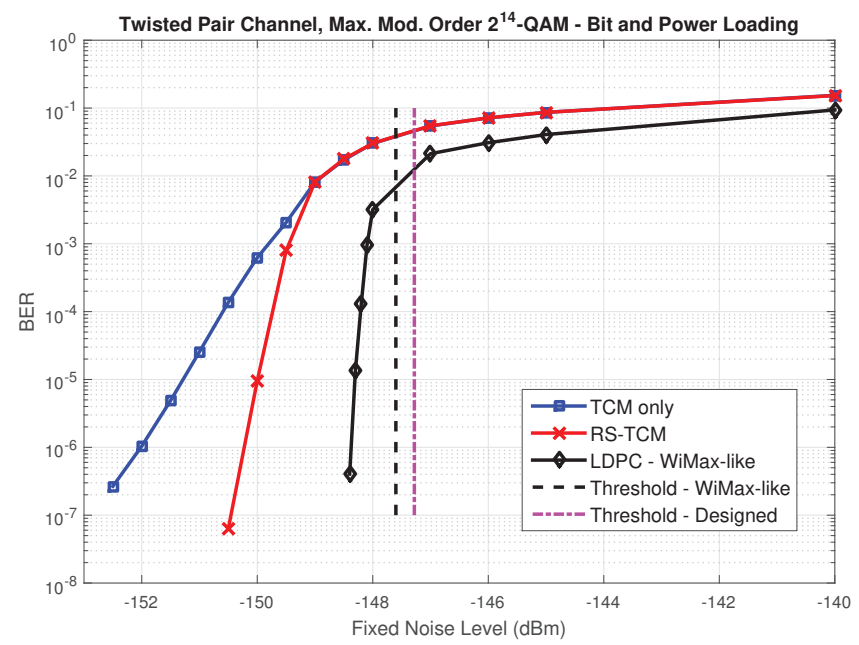

Fig. 9. Results for the $100 \mathrm{~m}$ line with bit- and power-loading, with maximum modulation order 14. Bit loading and coding rate for LDPC-BICM and RSTCM systems are the same, giving equal data rates.

\section{CONCLUSION}

In this paper, a joint design method for the LDPC code parameters and interleaver of a BICM system for use on DSL channels was proposed. Through a simulation study, LDPC coded BICM was demonstrated to offer significant gain over the legacy coding scheme currently used in DSL systems. The proposed method was demonstrated to achieve consistent gains over the already capacity approaching LDPC codes, through a number of scenarios.

It should be noted that, given the fixed attenuation properties of the twisted pair channel and the slowly varying nature of the noise encountered, the proposed method may be employed with little to no additional on-line computational cost, i.e., the extra effort required to realise the gain offered is incurred only at the parameter design stage. Additionally, the proposed approach may easily be extended to other channels and modulation schemes. In future work, the bit channel based threshold analysis may allow a more accurate choice of the values $G$ and $\Gamma$ in channel loading, to offer data rate gains in the system.

\section{ACKNOWLEDGEMENT}

This work was supported by EPSRC under project EP/N004299/1, Cooperative Backhaul Aided Next-Generation Digital Subscriber Loops. Additional work was supported by Royal Society Grant IF170002. The authors thank the Research Council and Royal Society for this funding.

\section{REFERENCES}

[1] M. Timmers, M. Guenach, C. Nuzman, and J. Maes, "G.fast: evolving the copper access network," IEEE Communications Magazine, vol. 51, no. 8, pp. 74-79, August 2013.

[2] J. Maes and C. J. Nuzman, "The past, present, and future of copper access," Bell Labs Technical Journal, vol. 20, pp. 1-10, 2015.

[3] ITU-T Recommendation G.9701, "Fast access to subscriber terminals (g.fast) - physical layer specification," Standard.

[4] M. A. Ahmed, C. T. Healy, A. F. A. Rawi, and C. C. Tsimenidis, "Bidirectional beamforming bit error ratio analysis for wireline backhaul networks," in 2016 24th European Signal Processing Conference (EUSIPCO), Aug 2016, pp. 295-299.

[5] G. Ginis and J. M. Cioffi, "Vectored transmission for digital subscriber line systems," IEEE Journal on Selected Areas in Communications, vol. 20, no. 5, pp. 1085-1104, Jun 2002.

[6] G. Caire, G. Taricco, and E. Biglieri, "Bit-interleaved coded modulation," in Proceedings of IEEE International Symposium on Information Theory, Jun 1997, pp. 96-.

[7] J. Hou, P. H. Siegel, L. B. Milstein, and H. D. Pfister, "Capacityapproaching bandwidth-efficient coded modulation schemes based on low-density parity-check codes," IEEE Transactions on Information Theory, vol. 49, no. 9, pp. 2141-2155, Sept 2003.

[8] S. ten Brink, G. Kramer, and A. Ashikhmin, "Design of low-density parity-check codes for modulation and detection," IEEE Transactions on Communications, vol. 52, no. 4, pp. 670-678, April 2004.

[9] N. von Deetzen and S. Sandberg, "Design of unequal error protection ldpc codes for higher order constellations," in 2007 IEEE International Conference on Communications, June 2007, pp. 926-931.

[10] B. Joshi and A. Thangaraj, "Exit chart based design of ldpc codes for higher order constellations," in 2013 National Conference on Cоттиnications (NCC), Feb 2013, pp. 1-5.

[11] K. Yan, T. Cheng, F. Yang, K. Peng, and J. Song, "Improved design of bit mapping based on exit-chart analysis for dvb-t2 system," IEEE Transactions on Consumer Electronics, vol. 57, no. 4, pp. 1579-1585, November 2011.

[12] G.9701 : Fast access to subscriber terminals (G.fast) - Physical layer specification, International Telecommunication Union (ITU) Std., 2014.

[13] L.-F. Wei, "Trellis-coded modulation with multidimensional constellations," IEEE Transactions on Information Theory, vol. 33, no. 4, pp. 483-501, Jul 1987.

[14] Y. Li and W. E. Ryan, "Bit-reliability mapping in ldpc-coded modulation systems," IEEE Communications Letters, vol. 9, no. 1, pp. 1-3, Jan 2005.

[15] P. K. Vitthaladevuni, M. S. Alouini, and J. C. Kieffer, "Exact ber computation for cross qam constellations," IEEE Transactions on Wireless Communications, vol. 4, no. 6, pp. 3039-3050, Nov 2005.

[16] T. J. Richardson, M. A. Shokrollahi, and R. L. Urbanke, "Design of capacity-approaching irregular low-density parity-check codes," IEEE Transactions on Information Theory, vol. 47, no. 2, pp. 619-637, Feb 2001.

[17] M. Yang, W. E. Ryan, and Y. Li, "Design of efficiently encodable moderate-length high-rate irregular ldpc codes," IEEE Transactions on Communications, vol. 52, no. 4, pp. 564-571, April 2004.

[18] "Ieee standard for local and metropolitan area networks part 16: Air interface for broadband wireless access systems," IEEE Std 802.16-2009 (Revision of IEEE Std 802.16-2004), pp. 1-2080, May 2009. 